\section{P184 VAGINAL MICROBIOTA ASSOCIATED WITH ONCOGENIC HPV IN A COHORT OF HPV-VACCINATED WOMEN LIVING WITH HIV}

${ }^{1}$ Elisabeth Mcclymont ${ }^{*},{ }^{2}$ Arianne Albert, ${ }^{3}$ François Coutlée, ${ }^{4}$ Marette Lee ${ }^{5}$ Sharon Walmsley, ${ }^{2}$ Nancy Lipsky, ${ }^{6}$ Janet Hill, ${ }^{7}$ Deborah Money. ${ }^{1}$ University of British Columbia, Obstetrics and Gynecology, Vancouver, Canada; ${ }^{2}$ Women's Health Research Institute, Vancouver, Canada; ${ }^{3}$ 'Université de Montréal, Microbiologie Médicale et Infectiologie, Montreal, Canada; ${ }^{4} B C$ Cancer, Vancouver, Canada; ${ }^{5}$ University Health Network, Toronto, Canada; 'University of Saskatchewan, Veterinary Microbiology, Saskatoon, Canada; ${ }^{7}$ University of British Columbia, Vancouver, Canada

\subsection{6/sextrans-2019-sti.338}

Background The role of the cervico-vaginal microbiome in the incidence and persistence of HPV infection is not well understood, particularly in the context of HIV infection. It is critical to understand this relationship in women living with HIV (WLWH) due to much higher rates of HPV-related disease in this population.

Methods WLWH were offered three doses of $\mathrm{qHPV}$ vaccine in a multi-centre study. Visits were at months $-3,0,2,6,12,18$, 24, and annually thereafter. Participants provided health data, HPV DNA samples, and cervico-vaginal swabs for microbiota sequencing (cpn60 amplicon). Persistent HPV was defined as the same HPV type in samples detected at $\geq 2$ consecutive visits.

Results 283 cervico-vaginal microbiota samples from 186 women were sequenced (1-3 samples/woman). Samples were taken between 3-8 years post-vaccination. Participants were predominantly Black (39.2\%) and Caucasian (37.1\%). At baseline, the median age was 38 years (range: 13-66, IQR: 3245), median CD4 count was 490 cells $/ \mathrm{mm}^{3}$ (IQR: 370-680), and $67.4 \%$ had an HIV viral load $<50$ copies $/ m L$. At the time of microbiota swab collection, median CD4 count was 619 (IQR: 409-794). Samples taken at the time of incident HPV detection $(n=44)$ displayed significantly higher relative abundance of Gardnerella vaginalis A than samples without incident HPV. Samples from women with persistent oncogenic HPV infection $(n=41)$ had greater relative abundances of Porphyromonas uenonis and Prevotella timonensis than samples without persistent HPV.

Conclusion This data supports previous reports of an association between Gardnerella vaginalis subtype A and HPV incidence. Porphyromonas uenonis and Prevotella timonensis should be further explored as potential co-factors in HPV persistence.

Disclosure No significant relationships.

\section{P186 NEISSERIA GONORRHOEAE INFECTIONS AMONG PEOPLE LIVING WITH HIV ON ART AT STI CLINIC IN KUMASI, GHANA}

\begin{abstract}
${ }^{1}$ Thomas Agyarko-Poku*, ${ }^{2}$ Kwame Buabeng, ${ }^{3}$ Alex Owusu Ofori, ${ }^{4}$ Yaw Adu Sarkodie. ${ }^{1}$ Suntreso Government Hospital, Ghana Health Service, Genitourinary Medicine, Kumasi, Ghana; ${ }^{2}$ Kwame Nkrumah University of Science and Technology, Kumasi Ghana; ${ }^{3}$ Department of Pharmacy Practice, Faculty of Pharmacy and Pharmaceutical Sciences, College of Health Sciences, Kumasi, Ghana; ${ }^{3}$ Kwame Nkrumah University of Science and Technology, Kumasi Ghana; ${ }^{2}$ School of Medicine and Dentistry Sciences, College of Health Sciences, Kumasi, Ghana; ${ }^{4}$ Kwame Nkrumah University of Science and Technology, Kumasi Ghana, School of Medicine and Dentistry Sciences, College of Health Sciences, Kumasi, Ghana
\end{abstract}

10.1136/sextrans-2019-sti.339

Background HIV infection is a risk factor for the acquisition and transmission of other sexually transmission infections
(STIs). Antiretroviral therapy (ART) suppresses viral load and generally improves the lives of people living with HIV (PLHIV) including resumption of sexual activities. This may lead to an increase in STI rates. We determined the prevalence of Neisseria gonorrhoeae among PLHIV who have been on ART

Methods This cross sectional study conducted among PLHIV between June and August 2018 employed a sensitive multiplex real time Polymerase chain reaction (PCR) assay that simultaneously detects the seven most common bacterial pathogens responsible for STI's. PLHIV attending a specialist STI clinic at Suntreso Government Hospital in Kumasi, Ghana who had been on antiretroviral therapy (ART) for more than 5 years were eligible for enrolment into the study. Informed consent was obtained prior to enrolment. Data was analysed using SPSS version 16 .

Results There were 400 PLHIV enrolled into the study consisting of $224(56.0 \%)$ males and $176(44.0 \%)$ females. Majority $324(81.1 \%)$ of the participants were asymptomatic. Overall, $245(61.3 \%)$ of enrolled patients were positive for at least one of the seven pathogens tested. Neisseria gonorrhoeae was the most common pathogen $44(10.1 \%)$ detected followed by Mycoplasma genitalium $(26 ; 6.4 \%)$ and Chlamydia trachomatis (5; $1.3 \%)$. Only $3.9 \%$ of the participants with gonorrhoeae had symptoms.

Conclusion Neisseria gonorrhoeae remains an important causative pathogen for STI in persons living with HIV. There is the likelihood of most of these infections going undetected since most of them did not have symptoms. Improved diagnostic methods like PCR are needed to identify and treat such STIs effectively when PLHIV who are on ART.

Disclosure No significant relationships.

\section{P187 ANAL CANCER AND PRECURSOR LESIONS IN HIV- INFECTED PERSONS: A CLINICAL COHORT STUDY}

${ }^{1}$ Yuanfan $\mathrm{Ye}^{*},{ }^{2} \mathrm{Greer}$ Burkholder, ${ }^{1}$ Howard Wiener, ${ }^{1}$ Amrita Mukherjee, ${ }^{2}$ Alexandra Gubbels, 'Sadeep Shrestha. 'University of Alabama at Birmingham, Epidemiology, Birmingham, USA; ${ }^{2}$ University of Alabama at Birmingham, Medicine, Birmingham, USA

\subsection{6/sextrans-2019-sti.340}

Background Infection with high-risk human papillomavirus increases the risk of developing anal cancer (AC). AC is rare in general populations (incidence rate: 1.8 cases per 100,000 each year). However, recent studies have seen an increased number of AC among people living with HIV-1 infection (PLWH). We aimed to investigate AC regarding its incidence rate and sociodemographic characteristics in a HIV clinical cohort in the southeastern United States.

Methods Electronic health records between 2006 and early 2018 from an academic HIV Clinic at the University of Alabama at Birmingham were reviewed retrospectively. The HIV clinic treats more than $30 \%$ of total PLWH in Alabama. Patients with at least two clinical visits and aged 18 and above at HIV diagnoses were included. Age at AC diagnosis, self-reported race and sexual orientation were tested in univariate analyses. All significant variables were fitted in a multivariable logistic model adjusting for nadir CD4 counts and highest viral load (VL) prior to AC diagnoses or last clinical visits for non-AC subjects. 
Results Among 4,484 PLWH (1632 whites, 2676 blacks, 176 other races; 1031 women, 3429 men, 24 transgenders) over a total follow-up of 22188 years, there were 43 ACs (all men, 24 whites, and 18 blacks). The median age at AC diagnoses was $52 ; 36$ were men who have sex with men (MSM) and 7 were heterosexual men $(\mathrm{P}<0.0001)$. The incidence was 267.2 cases per 100,000 men each year. Being male $(\mathrm{P}<0.0001)$, MSM $(P<0.0001)$, white $(p=0.03)$, aged $45-54$ years $(p=0.02)$, and with low nadir CD4 $(p<0.0001)$ were independently associated with AC.

Conclusion AC incidence among HIV-infected men was 148.4 times higher with earlier onset than general populations. However, public awareness of AC screening, specifically among the targeted population is lacking. This study can be valuable for the development of related screening programs and clinical practice guidelines for PLWH in the region.

Disclosure No significant relationships.

\section{P189 EVALUATION OF APTIMA HIV-1 QUANT ASSAY PERFORMANCE USING PLASMA AND DRIED BLOOD SPOTS}

Julie Nelson*_Kara Compliment, Paul Alabanza, Dana Lapple, Takesha Mcmillion, Marcia Hobbs. University of North Carolina at Chapel Hill, Microbiology and Immunology, Chapel Hill, USA

\subsection{6/sextrans-2019-sti.341}

Background The Hologic Aptima HIV-1 Quant Assay that is currently available on the Panther system is FDA-cleared for plasma viral load monitoring. We compared to other assays for use with dried blood spots (DBS) and for early infant diagnosis (EID) using plasma and DBS.

Methods Spiked dilution panels of plasma and DBS were made using negative donor blood and subtype B and subtype C HIV-1 stocks. Patient samples included plasma from HIV-1infected adults, plasma from HIV-1-infected infants and exposed uninfected infants, and DBS from infected adults. Samples were run on Aptima Quant and either Abbott RealTime HIV-1 Quant assay or Hologic Aptima HIV-1 Qual assay.

Results Aptima Quant and Abbott Quant were comparable: the $95 \%$ limit of detection was $42 \mathrm{cp} / \mathrm{ml}$ for Aptima Quant and $91 \mathrm{cp} / \mathrm{ml}$ for Abbott Quant. EID comparisons showed that Aptima Quant and Qual had 100\% sensitivity and 98.9\% specificity with overall agreement between the assays of $98.4 \%$. Aptima Qual had a slightly lower limit of detection $(95 \%$ limit of detection was $27 \mathrm{cp} / \mathrm{ml}$ for Aptima Qual and $65 \mathrm{cp} / \mathrm{ml}$ for Aptima Quant). With spiked DBS, all 16 DBS with $>30 \mathrm{cp} / \mathrm{ml}$ on Aptima Quant were detected on Aptima Qual, 10/12 (83\%) with '<30 detected' on Aptima Quant were detected on Aptima Qual, and 2 of 14 (14.3\%) with 'not detected' on Aptima Quant were detected on Aptima Qual. Among 200 DBS from infected adults, overall agreement between the Aptima Quant and Aptima Qual was 90\% when '<30 detected' was counted at positive. 13 DBS from uninfected adults were negative on both assays.

Conclusion The Hologic Aptima HIV-1 Quant assay performed similarly for viral load and EID on both plasma and DBS samples. Our data suggest the ' $<30$ detected' result could be used as the indeterminate range for Aptima Quant using DBS, as recommended by the new WHO guidelines.

Disclosure No significant relationships.

\section{P190 THE ROLE OF HELPLINE COUNSELING IN HIV STATUS DISCLOSURE AMONG SEXUAL PARTNERS: A CASE STUDY OF TOLL FREE HELPLINE IN UGANDA}

Julius Ssekinkuse*. Communication for Development Foundation Uganda, Counseling, P.O. Box 8734 Kampala, Uganda, Uganda

\subsection{6/sextrans-2019-sti.342}

Background Globally, 35.3 million people were living with HIV at the end of 2012. Sub-Saharan Africa remains most severely affected, with nearly 1 in every 20 adults living with HIV and accounting for $71 \%$ of the people living with HIV worldwide. Uganda has sustained some impressive response to HIV/AIDS epidemic grounded in a multi-sectoral approach coordinated by Uganda AIDS Commission. The response to stop and control HIV/AIDS has yielded many useful strategies and yet mistakes and missed opportunities. HIV/AIDS continues to be a major socio-economic challenge and is among the leading causes of morbidity and mortality given the increasing new infections due to status non-disclosure among sexual networks. Feedback from the Helpline indicate that those infected fear to disclose their HIV status to their sexual partners for many reasons thus the Helpline comes in to fill this gap through telephone counseling with timely responses Methods Data from Telephone calls received from those fearing to disclose their HIV status to their sexual partners was reviewed. A random selection of feedback responses was sampled to find out how the Helpline was helping the community deal to with the problem

Results Sampled 400 positive feed backs from community members who had benefited from Helpline counseling revealed that timely telephone counseling empowered them to disclose their HIV status to their sexual partners because of the prevention benefits discussed with Helpline counselors. The helpline empowered and educated callers about HIV and other STIs status disclosure benefits especially prevention of new infections

Conclusion The results of this study reveal that Helpline counseling can be a helpful resource in HIV/STIs prevention by empowering those infected to disclose their status with their sexual partners to protect and prevent new infections.

Disclosure No significant relationships.

\section{P191 SOUTH INDIAN LONG-DISTANCE TRUCK DRIVERS STILL AT HIGH-RISK FOR HIV/STI}

${ }^{1}$ Karl Krupp*, ${ }^{2}$ Kavitha Ravi, ${ }^{2}$ Anisa Khan, ${ }^{2}$ Vijaya Srinivas, ${ }^{2}$ Poornima Jayakrishna, ${ }^{2}$ Rishika Gupta, ${ }^{2}$ Neha Joshi, ${ }^{3}$ Purnima Madhivanan. ${ }^{1}$ Florida International Univeristy, Department of Health Promotion and Disease Prevention, Robert Stempel College of Public Health, Miami, USA; ${ }^{2}$ Public Health Research Institute of India, Prerana Women's Health Initiative, Mysore, Karnataka, India; ${ }^{3}$ Florida International University, Epidemiology, Miami, USA

\subsection{6/sextrans-2019-sti.343}

Background In a typical year, it is estimated that there are about 5 million long-distance truck drivers delivering goods on 3.3 million kilometers of roadways in India. Due to their high-risk behavior, truckers have long been considered a bridge population associated with the spread of sexually transmitted infection (STI) including HIV. The last national study of HIV prevalence in Indian truck drivers, completed in 2008, found an overall HIV prevalence of $4.6 \%$ and $2.7 \%$ for syphilis among long-distance truck drivers. 